\title{
ANÁLISIS COMPARATIVO TEMPORAL DE LOS PROCESOS DE RECONOCIMIENTO DE USOS NEOLÓGICOS FORMALES Y SEMÁNTICOS
}

\section{A TIME-COURSE COMPARATIVE ANALYSIS OF THE RECOGNITION PROCESSES OF FORMAL AND SEMANTIC NEOLOGICAL USES}

\author{
CARMen VARO VARO \\ Universidad de Cádiz \\ carmen.varo@uca.es
}

Enviado: $30 / 05 / 2019$

Aceptado: 03/09/2019

\begin{abstract}
Resumen
En este trabajo nuestro objetivo fundamental es explorar la delimitación entre neología formal y neología de significado, a través de un acercamiento experimental basado en el registro de los tiempos de decisión léxica. Para ello hemos tomado como referencia un conjunto de usos neológicos contextualizados, que representan las principales manifestaciones de ambos tipos. Los resultados de este estudio no sólo nos permiten comprobar las implicaciones de distintas estrategias cognitivas que repercuten en el modo de procesamiento, sino que además nos ayudan a trazar un mapa temporal general de los procesos de reconstrucción de la estructura semántica y formal de la palabra.

PALABRAS CLAVE: neología, neología formal, neología semántica, procesamiento léxico, lingüística aplicada.
\end{abstract}

\begin{abstract}
The aim of this work is to look into the distinction between formal neology and semantic neology through an experimental approach based on the recording of lexical decision times. For that purpose, we have taken as a reference a set of contextualized neological uses, which represent the main manifestations of both types. The results of this study not only allow us to verify the implications of different cognitive strategies that affect the way of processing, but also help us to draw a general time-course map which reflects the reconstructing processes of the semantic and formal structure of the word.

KEYWORDS: neology, formal neology, semantic neology, lexical processing, applied linguistics.
\end{abstract}

Para citar este artículo / To cite this article: Varo Varo, Carmen (2019). Análisis comparativo temporal de los procesos de reconocimiento de usos neológicos formales y semánticos. ELUA, 33: 195-209. doi: 10.14198/ELUA2019.33.10

Enlace / Link: http://dx.doi.org/10.14198/ELUA2019.33.10 


\section{LA DELIMITACIÓN ENTRE NEOLOGÍA FORMAL Y NEOLOGÍA SEMÁNTI- CA DESDE LA PERSPECTIVA NEUROCOGNITIVA}

La distinción entre neología de forma y neología de sentido, popularmente extendida en la bibliografía sobre creación léxica ${ }^{1}$, suele sustentarse en la priorización en la primera modalidad de la perspectiva de los significantes implicados y, en la segunda, de los significados que se ven sujetos a un reajuste. Frente a ello, la aproximación psicolingüística a este fenómeno proporciona una singular vía de caracterización, al ser los denominados usos neológicos abordados en el seno de los procesos del hablar en que son producidos y comprendidos, atendiendo a los mecanismos mentales que los sustentan (cf. Díaz Hormigo \& Varo Varo 2012). Paralelamente, nos proporciona suficientes herramientas para profundizar en la delimitación y definición particular de cada uno de sus diferentes tipos (cf. Varo Varo, Díaz Hormigo \& Paredes Duarte 2009).

En efecto, desde un enfoque neurocognitivo del léxico, en ambos casos puede decirse que interactúan una estructura de contenido, o basada en la activación de rasgos semántico-conceptuales, y una estructura de forma, o basada en la combinación de fonemas que pueden o no agruparse en morfemas. En este marco, partiendo de la posibilidad de reconstrucción del sistema léxico desde la perspectiva cerebral, se asume que el conocimiento lingüístico se organiza en forma de complejas redes neuronales interconectadas ${ }^{2}$, en las que la palabra no es sino la proyección de una doble estructura formal y semántica. Por otra parte, frente al clásico enfoque modular ${ }^{3}$ del léxico (cf. Fodor 1983), cada vez es más aceptada la idea de que durante el procesamiento lingüístico el individuo no opera con unidades prealmacenadas (cf. Lamb 1999: 375) y, como consecuencia, resulta inadecuado hablar de recuperación o acceso (cf. Bennett \& Hacker 2006: 44; Casasanto \& Lupyan 2015), sino que existe un mecanismo de reconstrucción léxica, vinculado a sistemas de análisis lingüístico y orientado por factores como la frecuencia y el contexto. Precisamente tal enfoque se adecúa a la perfección al fenómeno de la neología, pues da cuenta de la flexibilidad y el dinamismo característicos de los procesos de creatividad léxica. Además de ello, durante el procesamiento neológico adquiere particular protagonismo la habilidad cognitiva de la atención o prominencia (cf. Givòn 1983; Chafe 1994; Chiarcos, Barry \& Grabski 2011); entre otros), relacionada con los grados de activación de las estructuras conceptuales, y en la que participan procesos como la selección o habilidad para atender a partes de nuestra experiencia relevantes para un determinado propósito e ignorar aspectos irrelevantes para tal fin (cf. Croft \& Cruse 2004: 6). Si bien se

1 Para la delimitación entre neología de forma, formal u ordinaria frente a neología de sentido, de contenido o semántica (cf. Bastuji 1974; Pottier Navarro 1979). A estas dos modalidades otros autores añaden un tercer tipo, la neología por préstamo (cf. Auger \& Rousseau 2003).

2 Tales redes han sido descritas desde la perspectiva cerebral como conjuntos de neuronas, distribuidas sobre diversas regiones tanto de la corteza perisilviana como de otras áreas corticales distantes, dependientes entre sí y que trabajan formando distintas unidades funcionales (cf. Lamb 1999 y 2004; Pulvermüller 2001, 2005, 2012 y 2013; Kiefer \& Pulvermüller 2012, entre otros). Así pues, se descarta la interpretación tradicional de significado como centro unificado de contenido.

3 El enfoque modular impregna incluso los trabajos fundacionales de lingüística cognitiva, donde gramática y léxico forman parte de un mismo módulo donde se reconoce la existencia de un continuo desde palabras (o símbolos concretos) a oraciones (o símbolos esquemáticos) (cf. Langacker 1987). Frente a ello, se ha reconocido una relación directa entre asociacionismo y conexionismo, en cuanto desafío a la modularidad de los modelos tradicionales del lenguaje (cf. Brown 2006: 560). 
trata de una estrategia que, evidentemente, va más allá del ámbito del lenguaje, adquiere especial singularidad en el ámbito del discurso donde se incluyen los usos neológicos ${ }^{4}$.

Como uno de los principales referentes para la aproximación neurocognitiva al fenómeno de la neología semántica, debemos mencionar, en primer lugar, el creciente interés en las últimas décadas en el procesamiento de metáforas de nueva creación y su relación con el lenguaje literal. Así, en los trabajos iniciales desarrollados en esta dirección, basados en la lectura de enunciados con metáforas, frente a enunciados literales para medir la velocidad de producción, algunos autores no encontraron diferencias (cf. Ortony et alii 1978), si bien la ausencia de contraste temporal no implica que se usen distintos mecanismos de procesamiento. Por el contrario, otros estudios que hallaron ciertas diferencias temporales (cf. Janus \& Bever 1985) concluyeron que para acceder al significado metáforico es necesario acceder antes al contenido literal. Como vía de consenso, el modelo de inclusión de clases de Glucksberg propone la conexión entre la acepción de lengua y la metafórica basada en su vinculación polisémica (cf. Glucksberg, Gildea \& Bookin 1982; Glucksberg 2001 y 2003).

En segundo lugar, se ha atendido, desde una perspectiva psicolingüística, a aspectos como la interrelación de los procesos metafóricos, como fenómenos del lenguaje, con los diversos componentes de la cognición (cf. Gibbs 1994; Lakoff \&Johnson 1980). Asimismo, desde el enfoque de la neurociencia se ha estudiado especialmente la localización cerebral de tales procesos, mediante técnicas como la resonancia magnética funcional (RMf), la electroencefalografía para la obtención de potenciales evocados (EEG), etc. (cf. Geiger 1999; Arzouan et alii 2007; Mashal et alii 2007; Lai, Curran \& Menn 2009; Rapp, Mutschler \& Erb 2012; Benedek et alii 2014, entre otros). Como resultado, el procesamiento de metáforas de nueva creación ha sido descrito, en contraposición con el de metáforas convencionales y unidades léxicas de significado literal ${ }^{5}$, por la actuación de sistemas de análisis de naturaleza holista, que precisan del reclutamiento de mecanismos mentales y, por ende, áreas cerebrales ubicados fuera de los circuitos lingüísticos centrales, que incluyen el hemisferio derecho (cf. Yang 2012). De estos trabajos queremos resaltar, en relación con la neología, la priorización de los procesos de búsqueda semántica y de control ejecutivo y atención controlada que permiten inhibir el significado canónico o dominante e inciden en la recombinación de la información almacenada para construir nuevas creaciones léxicas ${ }^{6}$ (Silvia \& Beaty 2012).

En cambio, como posible marco de referencia para un acercamiento psicolingüístico a la neología formal, debemos resaltar aquellos estudios comparativos basados en la contraposición entre creaciones por derivación y pseudopalabras, desde donde se han abordado cuestiones como la representación de la morfología en el lexicón, la relación entre aspectos

4 La prominencia cognitiva ha sido frecuentemente abordada, en el marco de los modelos mentales relativos al lenguaje, como motor del control atencional o consciente, en particular en relación con la orientación y continuidad de los referentes a lo largo del discurso. Para una revisión del tema, cf. Gundel, Hedberg \& Zacharski 1993 o, más recientemente, Falk 2014.

5 En esta línea, se ha mostrado que, mientras que no hay diferencia en términos de duración temporal o esfuerzo cognitivo en el procesamiento de significados convencionales frente a significados metafóricos, los resultados son distintos para las metáforas de nueva creación (cf. Geiger 1999; Arzouan et alii 2007; Lai, Curran \& Menn 2009). 6 Las habilidades relacionadas con la fluidez verbal e intelectual, vinculadas a las funciones ejecutivas, justifican la relevancia de áreas como los giros frontales medio y superior izquierdos, además del córtex prefrontal dorsomedial y córtex cingulado posterior izquierdos, constatada a través de los datos más recientes de neuroimagen, para el procesamiento de metáforas de nueva creación (Benedek et alii 2014). 
morfológicos y aspectos léxico-semánticos de la palabra y las implicaciones de la facilitación semántica o formal (priming) y de los efectos contextuales y de frecuencia (cf. Burani \& Caramazza 19877; Cole, Beauvillain \& Segui 1989; Meunier \& Longtin 2007, entre otros). Efectivamente, se ha explorado el procesamiento de pseudopalabras, atendiendo a las diferencias entre las semánticamente interpretables y las semánticamente no interpretables, y entre las que siguen un patrón sistemático y las que no (Marslen-Wilson et alii 1994). Como es sabido, mientras que, en el caso de la neología semántica, unidades fonológicamente presentes en el lexicón se vinculan con significados que no se corresponden con el conjunto de contenidos asociados a cada entrada en cuestión, la neología formal atañe a unidades polimorfémicas cuya secuencia fonológica no existe como tal en el lexicón. La inexistencia previa de tales formas, no contemplada en los principales modelos explicativos del procesamiento léxico, al estar inspirados en la idea de recuperación, hace necesario profundizar en el papel del análisis morfológico no sólo desde el punto de vista de la descomposición formal sino también de la integración semántica entre morfemas.

Por consiguiente, puede mantenerse que las evidencias sobre cómo el cerebro integra anteriores conocimientos para producir nuevas ideas, junto al hallazgo de sustratos cerebrales compartidos por funciones lingüísticas y otras funciones cognitivas, en lo que a la neología se refiere, no sólo sirven de base explicativa de la separación entre los aspectos semánticos y formales del léxico, sino que también justifican las indudables implicaciones de mecanismos como la memoria y la atención en relación con nuestras múltiples experiencias sensoriales, motoras y emocionales ${ }^{8}$ desde el punto de vista del enriquecimiento contextual durante el proceso neológico. En suma, los numerosos datos experimentales aportados por la aproximación psicolingüística y los conocimientos neurológicos acerca de la organización cerebral del conocimiento léxico nos pueden deparar una explicación más afinada del fenómeno y de las diferentes modalidades de la neología.

Para profundizar en los procesos psicolingüísticos y las bases neurocognitivas que explican la producción e interpretación de las nuevas unidades, en este trabajo nuestros objetivos han sido: 1) analizar las estrategias cognitivas que repercuten en el procesamiento de usos neológicos en contextos, 2) explorar la diferencia entre neología formal y neología semántica y 3) trazar un mapa temporal general de los procesos de reconstrucción de la estructura semántica y formal de la palabra.

\section{APROXIMACIÓN TEMPORAL AL PROCESAMIENTO NEOLÓGICO}

Con el propósito de explorar la delimitación entre neología formal y neología semántica en el ámbito del procesamiento lingüístico, a través de un acercamiento experimental basado

7 El modelo de acceso dual de Burani y Caramazza (1987) ha supuesto una postura de consenso para superar la contraposición entre las principales vías explicativas del estatus de la morfología en el lexicón mental, la hipótesis de la lista exhaustiva, o estrategia basada en el morfema (cf. Taft \& Forster 1975), y la hipótesis de la lista exhaustiva, o estrategia que prioriza la palabra sobre el morfema (cf. Butterworth 1983 y 1989 y Dell \& O'Seaghdha 1992), pues contempla la activación en el sistema de acceso tanto de la palabra completa como de la raíz y sus afijos, que se propaga a otras unidades que comparten algún morfema.

8 Son cada vez más numerosos los modelos inspirados en representaciones conceptuales distribuidas y corporeizadas, en los que se atribuye mayor protagonismo a los sistemas sensorial, motor y emocional en la fundamentación de los procesos semánticos (cf. Kiefer \& Pulvermüller 2012). El problema que permanece sin aclarar es el de la delimitación del significado como contenido de lengua en relación con la esfera conceptual. 
en el registro de los tiempos de decisión léxica, hemos tomando como referencia algunos usos neológicos contextualizados, correspondientes a las principales manifestaciones de ambos tipos, que han sido tomados del Banco de neologismos que puede consultarse en el Centro Virtual Cervantes y es producto de una extracción y catalogación de las bases de datos de neologismos de BOBNEO del Observatori de Neologia del IULA. Como criterio de neologicidad se ha constatado, además, su actual ausencia en el diccionario académico.

\subsection{Metodología}

En particular, para la presentación de los estímulos utilizamos el software informático Eprime 3.0 (Psychology Software Tools). Este sistema permite la presentación computarizada de estímulos visuales y auditivos, así como el registro de los tiempos de reacción con una precisión de milisegundos ${ }^{9}$. En cuanto a los usos estudiados, en nuestro trabajo hemos partido de 10 enunciados donde se incluyen 6 usos neológicos formales y 4 semánticos (en adelante NF y NS). Entre los usos de NF, figuraban 2 casos de prefijación (NFP), 2 de sufijación (NFS) y 2 de composición (NFC). Por otra parte, hemos introducido otros 10 enunciados que responderían a una falsa neología formal (FNF) y una falsa neología semántica (FNS), en los que, en el primer caso, si bien se mantiene el entorno contextual, hemos sustituido los 6 NF por pseudopalabras cuasinónimas, y en el segundo caso hemos insertado las mismas unidades léxicas en contextos semánticamente inviables. Por último, se ha incluido 10 enunciados control (C).

Respecto al procedimiento, estos 30 enunciados, de similar extensión, fueron presentados visualmente (pantalla de ordenador) y de forma desordenada a un total de 35 estudiantes universitarios, todos ellos nativos de español y del área de Humanidades, que debían realizar de manera individual y en un entorno silencioso una tarea de decisión léxica sobre la coherencia semántica de tales usos ${ }^{10}$. Inicialmente, aunque no se aclaró de manera explícita qué es neología formal y qué es neología semántica, se presentaron y explicaron con detalle ejemplos de la tarea para familiarizar a los participantes. Debemos señalar que, aunque era deseable, no se incluyó un mayor número de casos para impedir el descenso del nivel atencional necesario en la prueba realizada.

\begin{tabular}{|c|}
\hline Ejemplo 1 presentado (Fuente: El País, 2016) \\
\hline $\begin{array}{l}\text { El pensamiento global lucha contra excepcionalismos. [Tiempo ilimitado de lectura] } \\
\rightarrow \text { [Al pulsar se pasa a la siguiente pantalla] } \\
\text { Excepcionalismos [Tiempo limitado de respuesta. Se muestran dos opciones] } \\
\text { Sí (tiene coherencia semántica) }\end{array}$ \\
\hline Ejemplo 2 presentado (Fuente: ejemplo propio) \\
\hline $\begin{array}{l}48 \text { migrantes mueren tras excepcionalismos en Túnez. } \\
\rightarrow \\
\text { Excepcionalismos } \\
\text { Sí } \quad \text { NO (no tiene coherencia semántica) }\end{array}$ \\
\hline
\end{tabular}

9 Debemos agradecer la colaboración en el diseño de este experimento de Javier González de la Rosa y Florencia Sanmarino, del Departamento de Psicología de la Universidad de Cádiz.

10 Aunque se han tomado en consideración aspectos como edad, sexo y dominancia manual, ninguno de ellos resultó relevante en cuanto a los resultados obtenidos en el experimento. 
En tanto que el tiempo de lectura de cada enunciado fue ilimitado ${ }^{11}$, una vez que cada participante pasaba a la siguiente pantalla donde se interrogaba por la coherencia semántica de determinado uso léxico extraído del contexto verbal antes leído, debía decidir pulsando la tecla correspondiente lo más rápidamente posible Sí o No. Los aspectos analizados fueron, pues:

1. Tiempo de respuesta (TR) en milisegundos (ms) por palabras y por tipos: desde que se muestra la palabra en cuestión hasta que pulsa Sí o No. Se suprimieron aquellas respuestas cuya latencia fue superior a 5 segundos, con objeto de descartar posibles dudas interpretativas del enunciado en su conjunto.

2. Porcentaje de errores $(\% \mathrm{E})$ o número de respuestas que no se ajustan a lo esperado.

\subsection{Ejemplos analizados}

Los ejemplos analizados, con representación de las principales categorías gramaticales (verbo, sustantivo y adjetivo), recogen los procesos morfológicos de prefijación, derivación y composición, en el caso de la neología formal, y contemplan entornos contextuales con facilitación anterior y facilitación posterior, en el caso de la neología semántica:

\begin{tabular}{|c|c|c|}
\hline Procedimiento & Neología formal (NF) & Falsa neología formall $(\mathrm{FNF})^{12}$ \\
\hline \multirow{2}{*}{ Prefijación } & sobreaforo & *sobrevolumen \\
\hline & intrafamiliar & *intracasera \\
\hline \multirow{2}{*}{ Sufijación } & precariado & *eventualado \\
\hline & ciclable & *vehiculizable \\
\hline \multirow{2}{*}{ Composición } & geolocalizar & *geoponer \\
\hline & bioartificial & *biodisfrazado \\
\hline Procedimiento & \multicolumn{2}{|c|}{ Neología semántica (NS) / Falsa neología semántica (FNS) } \\
\hline Facilitación anterior & \multicolumn{2}{|c|}{$\begin{array}{c}\text { centrifugar } \\
\text { oceánica }\end{array}$} \\
\hline $\begin{array}{l}\text { Facilitación } \\
\text { posterior }\end{array}$ & \multicolumn{2}{|c|}{$\begin{array}{c}\text { cortafuegos } \\
\text { marciano }\end{array}$} \\
\hline
\end{tabular}

11 Aunque también se registró el tiempo total de la lectura de cada enunciado (hasta pasar a la siguiente pantalla), dado que no se solicitó a los participantes que se hiciera de manera veloz, no se ha considerado en este trabajo. 12 Respecto a la construcción de los falsos usos de NF aquí considerados, se ha optado por elementos léxicos de alta frecuencia en español (volumen, casero, poner, disfrazado), que pueden funcionar como cuasinónimos de los elementos léxicos presentes en los auténticos usos seleccionados. Nuestra intención fue observar, además de la relevancia de la familiaridad con cada uno de los componentes de estas formaciones, la repercusión de la frecuencia combinatoria de los elementos implicados. 


\section{Neología formal (NF) / Falsa neología formal (FNF).}

\section{Prefijación $^{13}$}

- El imputado es plenamente consciente de que existe un inmenso sobreaforo, que es responsabilidad de él mismo (...). (Fuente: El País, 2016)

/ *sobrevolumen

- Más de 57.000 mujeres víctimas de violencia intrafamiliar, con un promedio de 43 reportes diarios. (Fuente: El País, 2016)

/ *intracasero

\section{Sufijación}

- Ha sido una catástrofe arrastrar a la clase media al precariado. El conflicto ya no es entre clases, sino de cada uno con la sociedad. (Fuente: El País, 2016)

/ *eventualado

- Las actuaciones relacionadas con la bici se completarán con la ejecución de una via ciclable entre la avenida Diagonal y Esplugues de Llobregat. (Fuente: La Vanguardia, 2016)

/ *vehiculizable

\section{Composición}

- Por ahora, la web permite geolocalizar al usuario y a partir de ahí dispara todos los productos de los 30 comercios más cercanos. (Fuente: La Nación, 2016)

/ *geoponer

- Fabrican e implantan por vez primera vasos sanguíneos 'bioartificiales' en un modelo animal. (Fuente: $A B C, 2016$ )

/ *biodisfrazados

\section{Neología semántica con facilitación anterior (NSFA). Neología semántica con facilitación posterior (NSFP).}

- El ministerio debe asumir sus responsabilidades, no puede seguir centrifugándolas contra administraciones subestatales. (Fuente: El País, 2016)

- González madruga, pasea por cubierta y no cesa de dar instrucciones a la tripulación. Consignas envueltas en el celofán de su oceánica retórica. (Fuente: La Vanguardia, 2016)

- Tusk defendió la Unión Europea como el principal cortafuegos contra los conflictos en Europa. (Fuente: El Pais, 2016)

- Me parece un elemento un poco marciano que el presupuesto público de un estado no tenga posiciones politicas. (Fuente: Corpus oral, 2016)

13 Los casos de prefijación elegidos procuran evitar la confluencia con casos de pseudoprefijación (re-, in-, $a$-), que puedan ralentizar el proceso de acceso léxico, en lugar de facilitarlo. 
Falsa neología semántica con facilitación anterior (FNSFA) y con facilitación posterior (FNSFP). Noticias de prensa, ligeramente modificadas.

- Los líderes de Corea del Norte y del Sur se comprometen a firmar la paz y trabajar para centrifugar la península.

- La organización buscaba no solo terminar con la discriminación que prohibía a las mujeres su derecho oceánico a elegir un gobierno.

- En noviembre de 2017, un equipo de reputados científicos consiguió un marciano permiso que nunca antes había sido concedido.

- Un anuncio, publicado en un periódico francés, invitaba a los lectores a participar en un cortafuegos para recibir su horóscopo personalizado.

\subsection{Hipótesis exploradas}

Respecto a los presupuestos de partida para afrontar este estudio, de acuerdo con investigaciones previas sobre procesamiento de enunciados donde se incluyen metáforas o pseudopalabras, consideramos los siguientes:

1) Mayor tiempo para la neología formal frente a la neología semántica, por afectar ésta última a entidades léxicas familiares para el interpretante, al menos desde la perspectiva de su significante y por converger en la primera procesos morfológicos y semánticos

2) Mayor tiempo para las falsas neologías formal y semántica, por la falta de expectativa que generan y la mayor complejidad que implican.

3) Mayor tiempo para la neología semántica con facilitación posterior, donde el contexto previo no puede contribuir al reconocimiento léxico

4) Mayor tiempo para la neología formal por sufijación que para la neología formal por prefijación, si asumimos la facilitación del reconocimiento de componentes morfológicos durante el procesamiento.

\subsection{Datos obtenidos}

El promedio de TR, mostrado en figura 1, para los casos de NS fue de $1.480 \mathrm{~ms}$, con un rango de dispersión de respuestas relativamente bajo de $260 \mathrm{~ms}$. El menor TR registrado correspondió a cortafuegos, con $1.249 \mathrm{~ms}$. frente al mayor TR de $1.890 \mathrm{~ms}$ de oceánico. Respecto a la comparación entre casos de NS con facilitación anterior (centrifugar y oceánico) y casos con facilitación posterior (cortafuegos y marciano), los resultados obtenidos fueron muy similares, $1.482 \mathrm{~ms}$ para NSFA y $1.488 \mathrm{~ms}$ para NSFP, lo que descarta la aceleración de los procesos de preactivación semántica promovidos por el contexto. 


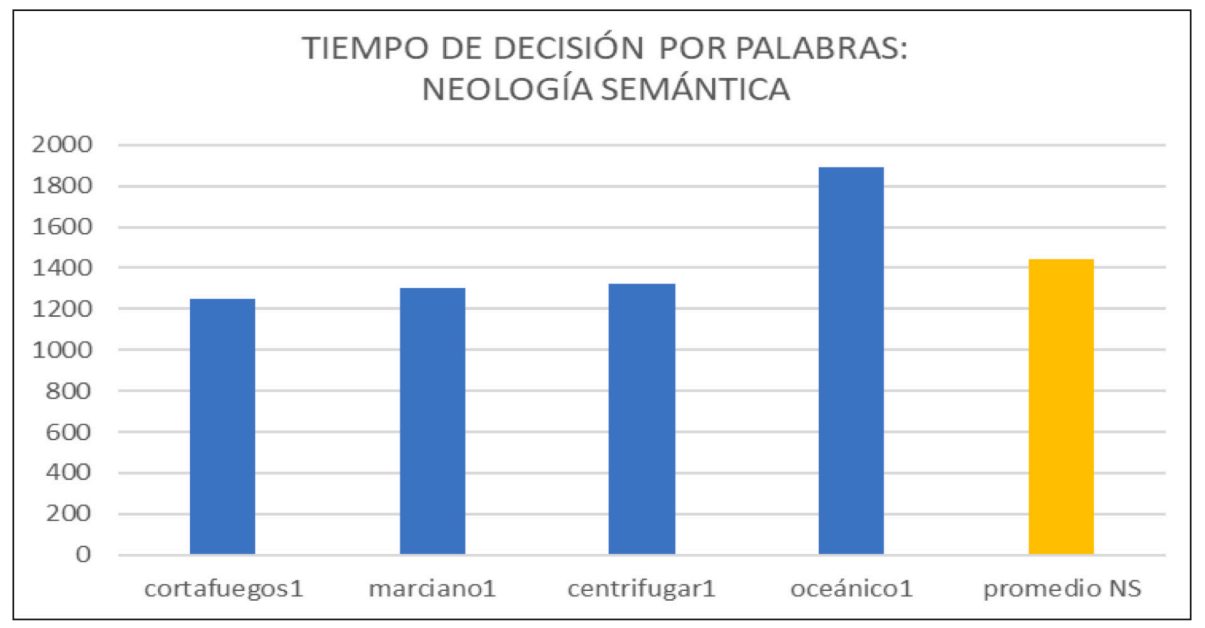

Figura 1

De la comparación entre NS y FNS, representada en figura 2, se observa que el TR es inferior para FNS en todos los ejemplos analizados. Este hecho parece apuntar hacia la intervención de pistas en el entorno sintagmático que precipitan el descarte en estos casos.

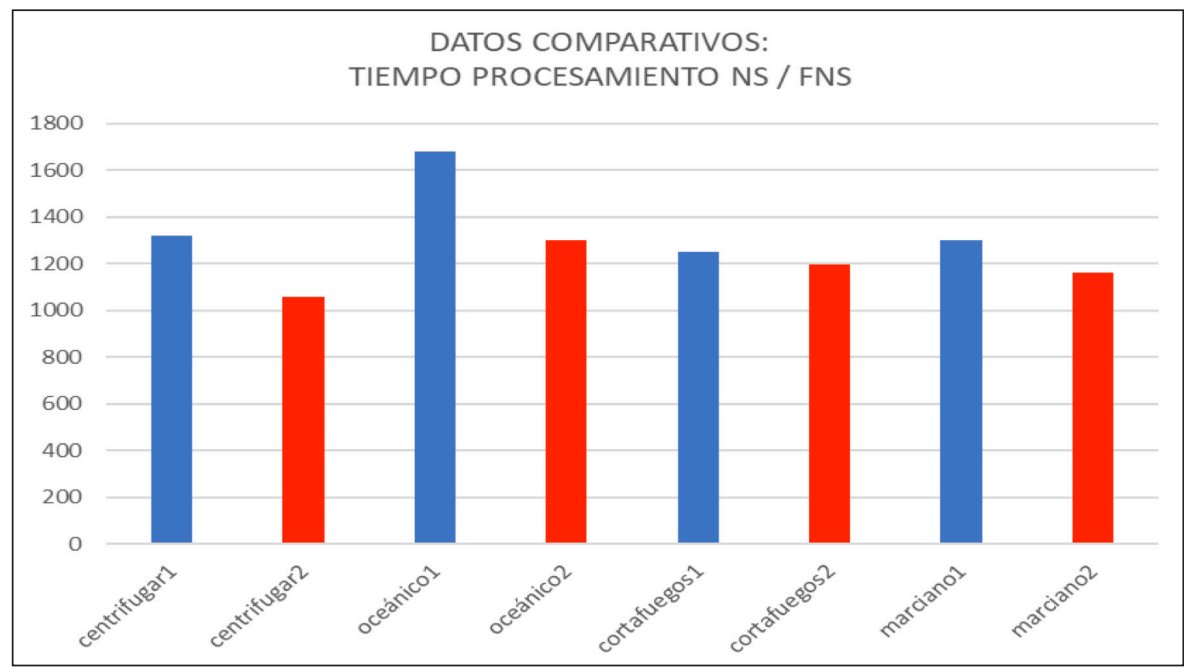

Figura 2

En los ejemplos analizados de NF, mostrados en figura 3, el rango de dispersión respecto a NS fue, incluso, menor, $156 \mathrm{~ms}$. Los TR se sitúan entre $1.073 \mathrm{~ms}$ de geolocalizar y 1.584 ms de ciclable, con un promedio de $1.326 \mathrm{~ms}$. Así pues, TR es en conjunto inferior al de NS. En cuanto a las modalidades de NF analizadas, se han hallado valores muy similares en el TR de prefijación $(1.477 \mathrm{~ms})$ y sufijación $(1.407 \mathrm{~ms})$. Parece, pues, que la respuesta no 
se ve beneficiada, al menos en los casos aquí considerados, por la identificación del prefijo, de la misma manera que en NS el TR tampoco era inferior en contextos con facilitación anterior. En cambio, el procedimiento de la composición muestra, frente a los de derivación, un TR menor (1.190 ms), lo que puede relacionarse con la mayor transparencia del primero.

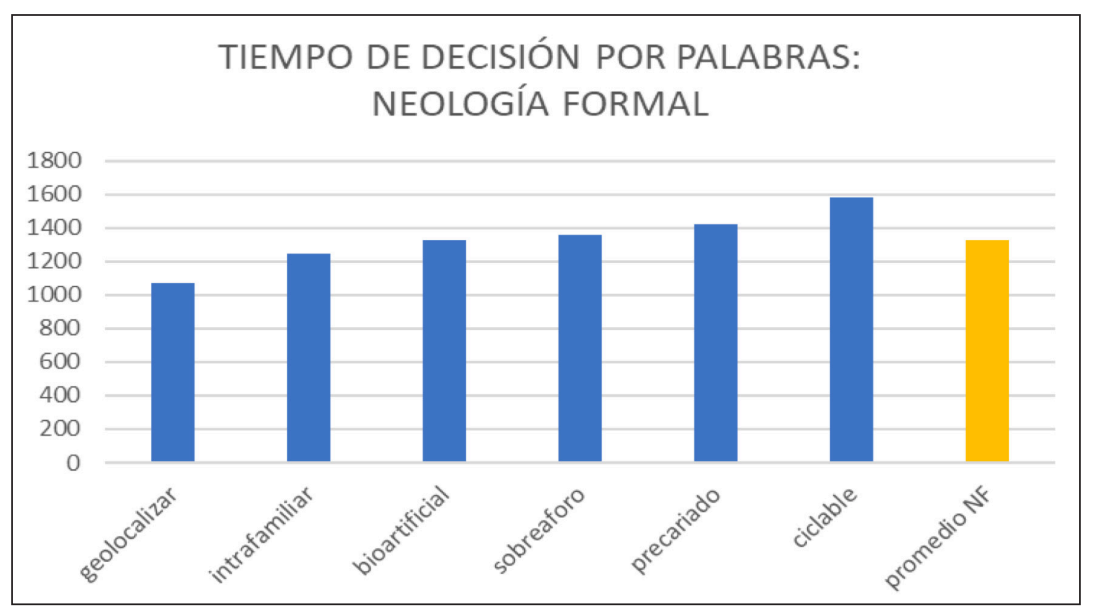

Figura 3

Como se muestra en figura 4, de forma semejante al caso de FNS, el TR es, en todos los casos analizados, inferior para FNF, dato que descarta la mayor o menor complejidad cognitiva de la unidad en cuestión como base principal para explicar la velocidad de procesamiento lingüístico.

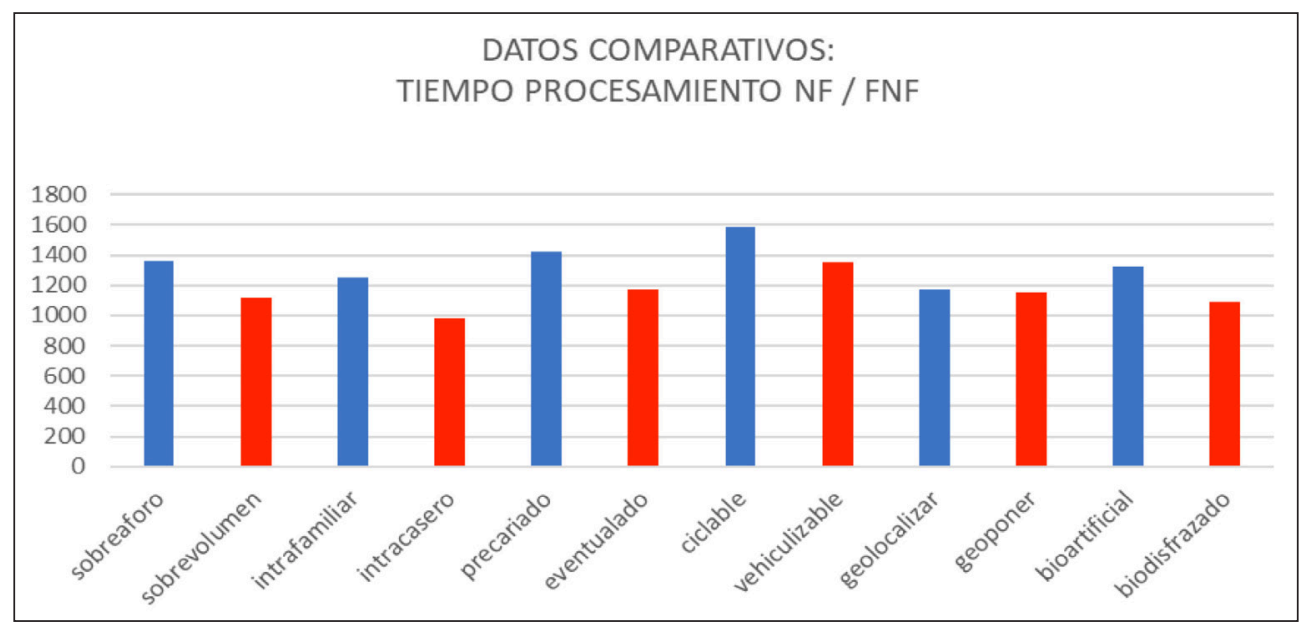

Figura 4

Registramos, por último, el porcentaje de errores para cada modalidad estudiada (véase figura 5). En este sentido, destaca NS con una cifra de casi el 39\%, frente al $16 \%$ de NF. 
Para las falsas NS y NF siempre se registra menor número de errores, lo que refuerza la validez de la prueba. El porcentaje de errores para los enunciados control (C), inferior al $1 \%$, otorga fiabilidad a los resultados obtenidos.

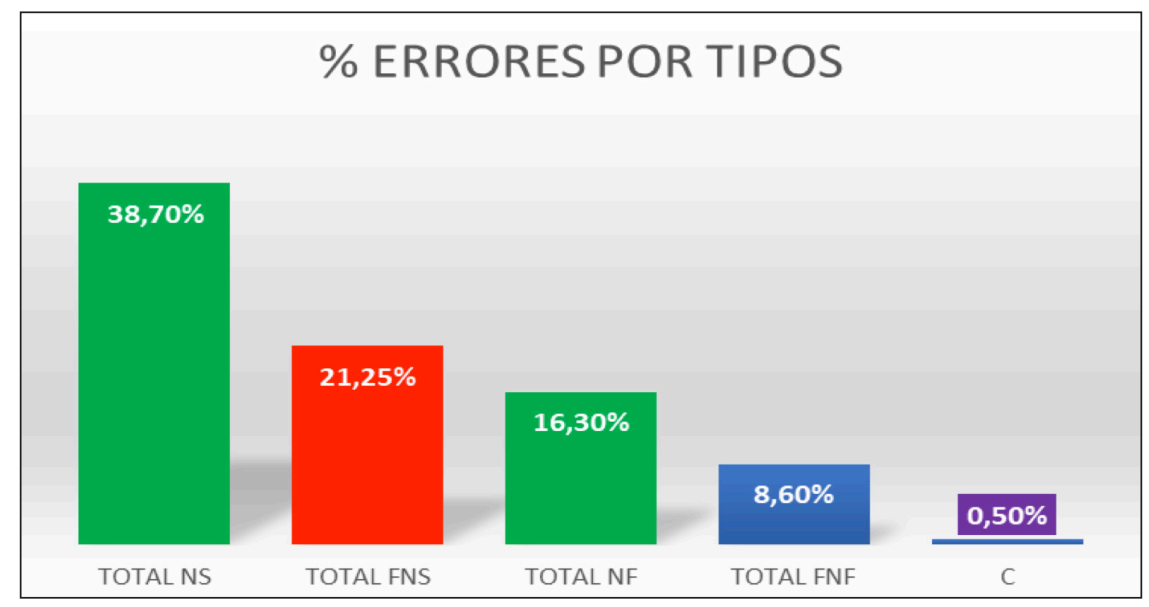

Figura 5

\section{ANÁLISIS DE LOS RESULTADOS}

Desde una perspectiva general, los resultados obtenidos tras este estudio exploratorio observacional permiten, de entrada, cuestionar aspectos como la complejidad del estímulo o la familiaridad de éste para dar cuenta del aumento o disminución del tiempo de respuesta y, por ende, del esfuerzo cognitivo invertido; antes bien, la confluencia de procesos morfológicos y semánticos en el caso de la neología formal, lejos de ralentizar el procesamiento léxico, más bien parece facilitarlo. Por el contrario, según se desprende tanto de los datos sobre TR como del número de errores registrados, la familiaridad, al menos con el significante, de la neología semántica no agiliza siempre la interpretación del contenido. Es evidente que para alcanzar conclusiones más sólidas sería deseable ampliar el corpus de estudio, con mayor número de unidades neológicas y contextos, e incrementar la muestra de población tomada como referencia. En todo caso, la aplicación de una tarea de decisión sobre la coherencia semántica de distintos enunciados nos ha permitido obtener datos temporales que precisamente sirven para valorar de forma objetiva los procesos de reconstrucción léxica. Además, la validez de los datos se ve refrendada por la escasa dispersión de las respuestas obtenidas entre informantes. Así pues, como derivación central de nuestro acercamiento, estos resultados ofrecen un punto de partida para explorar un nuevo enfoque del procesamiento léxico, si bien, requieren de mayor número de estudios para confirmarse.

En primer lugar, en cuanto a los datos temporales de decisión léxica, de acuerdo con estudios anteriores sobre procesamiento de pseudopalabras (cf., por ejemplo, Burani, Dovetto, Spuntarelli \& Thornton 1999; Burani, Arduino \& Marcolini 2006) y creaciones metafóricas con diferente grado de contextualización (cf. Gildea \& Glucksberg 1983), 
hemos podido constatar, a través de datos temporales, la relevancia del marco lingüístico donde se insertan los usos neológicos ${ }^{14}$. Ello nos permite redundar en la diferencia entre procesar palabras y procesar enunciados, así como la competición entre enriquecimiento contextual y procesos atencionales. De cualquier forma, somos conscientes de que los resultados obtenidos responden en parte a la presión impuesta por la limitación de tiempo en la respuesta.

En lo que respecta a la comparación entre NS y NF, no se cumple la expectativa de que NF se procese con mayor dificultad, pese a que en ella se combinan procesos morfológicos y semánticos simultáneamente. Recordemos que responderían a pseudopalabras no registradas en el lexicón mental. En este sentido, el menor tiempo de reconocimiento en NF indica que su interpretación se ve beneficiada por la confluencia de procesos de enriquecimiento semántico y de análisis morfológico. Asimismo, NF no precisa tanto del contexto como NS, si bien igualmente se beneficia de él. Sin embargo, en cuanto a la comparación de NF con FNF, la primera requiere mayor esfuerzo de procesamiento, probablemente por el conflicto generado entre la familiaridad con los componentes y la no familiaridad con la combinación, que permite excluir con mayor rapidez la FNF. Esta diferencia no es tan acusada como la que se observa entre NS y FNS. Así pues, de nuevo a través de estos datos se pone de manifiesto que el análisis morfológico no ralentiza el procesamiento, sino que más bien lo facilita.

De la comparación de NS con FNS se extrae, además del mayor tiempo de respuesta en NS, un incremento del número de errores o dudas, pues los falsos usos son descartados con mayor facilidad atendiendo a una posible estrategia de encaje contextual, que parece orientar todo el proceso de activación de la red semántica-conceptual, determinando así la inhibición de ciertas conexiones, que condicionan el tiempo de procesamiento y, como consecuencia, el esfuerzo cognitivo.

Si atendemos a los datos relativos a la influencia del entorno contextual, previo y posterior, en el caso de NS, y de la estructura derivativa, por prefijación o sufijación, en el caso de NF, observamos que la facilitación anterior no acelera el tiempo de respuesta, pues los valores son similares al caso de la facilitación posterior. Incluso, en los ejemplos analizados el número de errores registrados para el caso de la facilitación anterior ha sido ligeramente superior. Parece, pues, que la reconstrucción semántica a través de la raíz prima sobre la activación de asociaciones mediatizadas por la prefijación. Estos datos, sin duda, restan relevancia a la hipótesis lineal del procesamiento lingüístico y apuntan hacia una interpretación más dinámica y abierta del reconocimiento léxico.

\section{CONCLUSIONES}

Nuestra propuesta parte del significado como base de toda comunicación y en el que se recoge el conjunto de mecanismos mentales y factores que garantizan su eficacia en la comunicación cotidiana. Así, aunque los usos neológicos formales se comportan como una suerte de pseudopalabras, su interpretación concierne tanto a la estructura semántica

14 Estos resultados se alinean, por tanto, con estudios basados en ERP para medir N400 tras la escucha de elementos léxicos literales, metáforas familiares y metáforas nuevas en contextos relevantes e irrelevantes de distinto grado de complejidad, en los que se pone de relieve la relevancia del entorno contextual en la interpretación semántica (Pynte et alii 1996). 
subléxica como al contexto léxico inmediato. Por su parte, como se ha visto, la neología semántica afecta a unidades familiares desde el punto de vista de su configuración formal, pero cuyo contenido semántico requiere un amplio proceso de reconstrucción sémica en gran medida potenciado y, a veces, ampliado por el entorno contextual. En este punto, los datos aquí registrados se alejan de la idea de mera recuperación habitual en los modelos clásicos del procesamiento léxico.

A la luz de los resultados obtenidos tras este estudio, puede vislumbrarse una estrategia de enriquecimiento contextual, ya señalada por trabajos previos sobre el comportamiento de pseudopalabras y metáforas,a la que se agrega una estrategia de encaje sintagmático, que justifica los TR inferiores tanto para FNF como para FNS. Además, los datos comparativos que ilustran el papel de la facilitación en los marcos léxico y subléxico, confrontando la NS con facilitación anterior con la NS con facilitación posterior, de un lado, y la NF por prefijación con la NF por sufijación, de otro, restan relevancia a tales delimitaciones tipológicas, al menos, desde el enfoque psicolingüístico y, en general, a la interpretación lineal del procesamiento léxico, y ponen de manifiesto la actuación de una estrategia de procesamiento en paralelo de los significados. Como se ha tratado de mostrar, la consideración desde un prisma psicolingüístico, al estar los usos neológicos involucrados en los procesos de codificación y descodificación lingüísticas, hace posible alcanzar un nuevo enfoque interpretativo del significado léxico, más allá del análisis de las unidades aisladas. Por último, queremos resaltar el hallazgo de evidencias que apoyan la existencia de cooperación de los analizadores semántico y morfológico. Sólo desde ese punto de vista se concibe la reducción del tiempo de procesamiento y de errores en todos los casos analizados de NF, respecto a los de NS, como también el mayor rango de dispersión en NS frente a NF, resultantes de la acotación y orientación interpretativa impuesta por la estructura formal de la palabra a los múltiples procesos semánticos asociativos comunes a la creación léxica.

\section{REFERENCIAS BIBLIOGRÁFICAS}

Arzouan, Y. et alii (2007). "Dynamics of hemisphere activity during metaphor comprehension: Electrophisiological measures", NeuroImage, 36, pp. 222-251.

Auger, P. \& L. J. Rousseau (2003): Metodología de la investigación terminológica. Málaga: Universidad de Málaga.

Bastuji, J. (1974). “Aspects de la néologie sémantique”, La néologie lexicale. Langages, 36, pp. 6-19. Benedek, M. et alii (2014). "Creating metaphors: The neural basis of figurative language production", NeuroImage, 90, pp. 99-106.

Bennett, M. R. \& P. M. S. Hacker (2006). "Language and cortical function: Conceptual developments", Progress in Neurobiology, 80, pp. 20-52.

Brown, K. (ed.) $\left(2006,2^{\text {nd }}\right.$ ed.). Encyclopedia of Language and Linguistics. Amsterdam / London: Elservier.

Burani, C., Arduino, L. S. \& S. Marcolini (2006). "Naming Morphologically Complex Pseudowords: A headstart for the root?", The Mental Lexicon: a Journal of cross-disciplinary research,1, pp. 299-327.

Burani, C. \& A. Caramazza (1987). "Representation and processing of derived words", Language and Cognitive Processes, 2, pp. 217-227.

Burani, C., Dovetto, F. M., Spuntarelli, A., \& A. M. Thornton (1999). "Morpholexical access and naming: The semantic interpretability of new root-suffix combinations", Brain and Language, 68, pp. 333-339. 
Butterworth, B. (1983). "Lexical representation". En Butterworth, B. (ed.). Language production, Vol. 2. London: Academic Press, pp. 257-294.

Butterworth, B. (1989). "Lexical access in speech production”. En Marslen-Wilson, W. (ed.). Lexical representation and process. Cambridge, MA: MIT Press, pp. 108-135.

Casasanto, D. \& G. Lupyan (2015). "All concepts are ad hoc". En Margolis, E. \& S. Laurence (eds.). The Conceptual Mind: New directions in the Study of Concepts. Cambrigde MA: MIT Press, pp. 543-566.

Chafe, W. (1994). Discourse, consciousness, and time: The flow and displacement of conscious experience in speaking and writing. Chicago: The University of Chicago Press.

Chiarcos, C., Barry, C. \& M. Grabski (eds) (2011). Salience: Multidisciplinary perspectives on its function in discourse. New York / Berlin: de Gruyter.

Colé, P., Beauvillain, C. \& J. Segui (1989). "On the Representation and Processing of Prefixed and Suffixed Derived Words. A Differential Frequency Effect", Journal of Memory and Language, 28 , pp. 1-13.

Croft, W. \& D. A. Cruse (2004). Cognitive Linguistics. Cambridge: Cambridge University Press.

Dell, G. \& P. O' Seaghdha (1992). "Stages of lexical access in language production", Cognition, 42, pp. 287-314.

Díaz Hormigo, M ${ }^{\mathrm{a}}$ T. \& C. Varo Varo (2012). "Neology and Cognition". Linguistics Insights. Vol. 158. Eizaga Rebollar, B. (ed.). Studies in Linguistics and Cognition. Bern: Peter Lang, pp.15-34.

Falk, S. (2014). "On the notion of salience in spoken discourse - prominence cues shapping discourse structure and comprehension”, Travaux interdisciplinaires sur la parole et la langage, 30, pp. 1-23.

Fodor, J. A. (1983). The modularity of mind: An essay on faculty psychology. Cambridge MA, MIT Press.

Geiger, O. (1999). "Metaphors and the Mental Lexicon", Brain and Language, 68, pp. 190-198.

Gibbs, R. W. (1994). The Poetics of Mind: Figurative Thought, Language, and Understanding. Cambridge: Cambridge University Press.

Gildea, P. \& S. Glucksberg (1983). "On Understanding Metaphor. The Role of Context", Journal of Verbal Learning and Verbal Behavior, 22, pp. 577-590.

Givòn, T. (ed) (1983). Topic continuity in discourse: A quantitative cross-language study. Amsterdam: John Benjamins.

Glucksberg, S. (2001). Understanding Figurative Language: From Metaphors to Idioms. Oxford: Oxford University Press.

Glucksberg, S. (2003). "The psycholinguistics of metaphor", Trends in Cognitive Science, 7, pp. 9296.

Glucksberg, S., Gildea, P. \& H. A. Bookin (1982). "On understanding nonliteral speech: Can people ignore metaphors?", Journal of Verbal Learning and Verbal Behavior, 21, pp. 85-98.

Gundel, J. K., Hedberg, N. \& R. Zacharski (1993). "Cognitive status and the form of referring expressions in discourse", Language, 69, pp. 274-307.

Janus, R. A., \& T. G. Bever (1985). "Processing of metaphoric language: An investigation of the threestage model of metaphor comprehension", Journal of Psycholinguistic Research, 14, pp. 473-487.

Kiefer, M. \& F. Pulvermüller (2012). "Conceptual representations in mind and brain: theoretical developments, current evidence and future directions", Cortex, 48, pp. 805-25.

Lai, V., Curran, T. \& L. Menn (2009). "Comprehending conventional and novel metaphors: an ERP study”, Brain Research, 1284, pp. 145-155.

Lakoff, G. \& M. Johnson (1980). Metaphors We Live by. Chicago: University of Chicago Press.

Lamb, S. (1999). Pathways of the brain. The neurocognitive basis of language. Amsterdam / Philadelphia: John Benjamins.

Langacker, R. (1987). Foundations of Cognitive Grammar: Theoretical Prerequisites. I. Standford: Standford University Press.

Marslen-Wilson, W. et alii (1994). "Morphology and meaning in the mental lexicon", Psychological Review, 101,1, pp. 3-33. 
Mashal, N. et alii (2007). "An fMRI investigation of the neural correlates underlying the processing of novel metaphoric expressions", Brain and language, 100, pp. 115-126.

Meunier, F. \& C-M. Longtin (2007). "Morphological decomposition and semantic integration in word processing", Journal of Memory and Language, 56, pp. 457-471.

Ortony, A. et alii (1978). "Interpreting metaphors and idioms: Some effects of context on comprehension”, Journal of Verbal Learning and Verbal Behaviour, 17, pp. 465-477.

Pottier Navarro, H. (1979). "La néologie en espagnol contemporain”, Les langues néolatines, 229-230, pp. 148-172.

Pulvermüller, F. (2001). "Brain reflections of words and their meanings", Trends in Cognitive Sciences, 5, 12, pp. 217-24.

Pulvermüller, F. (2005). The neuroscience of language. Reimpr. Cambridge: Cambridge University Press.

Pulvermüller, F. (2012). "Meaning and the brain: the neurosemantics of referential, interactive, and combinatorial knowledge", Journal of Neurolinguistics, 25, pp. 423-59.

Pulvermüller, F. (2013). "How neurons make meaning: brain mechanisms for embodied and abstractsymbolic semantics", Trends in Cognitive Sciences, 17, 9, pp. 458-70.

Pynte, J. et alii (1996). "The Time-Course of Metaphor Comprehension: An Event-Related Potential Study", Brain and Language, 55, pp. 293-316.

Rapp, A. M., Mutschler, D. E. \& M. Erb (2012). "Where in the brain is nonliteral language? A coordinate-based meta-analysis of functional magnetic resonance imaging studies", NeuroImage, 63, pp. 600-610.

Silvia, P. J. \& R. E. Beaty (2012). "Making creative metaphors: the importance of fluid intelligence for creative thought", Intelligence, 40, pp. 343-351.

Taft, M. \& K. Forster (1975). "Lexical storage and retrieval of prefixed words", Journal of Verbal Learning and Verbal Behaviour, 14, pp. 638-647.

Varo Varo, C, Díaz Hormigo, M ${ }^{\mathrm{a}}$ T. \& M M J. Paredes Duarte (2009): "Modelos comunicativos y producción e interpretación neológicas", Revista de Investigación Lingüística, 12, pp. 185-216.

Yang, J. (2012). "The role of the right hemisphere in metaphor comprehension: a metaanalysis of functional magnetic resonance imaging studies", Human Brain Mapping, 35, 1, pp. 107-122. 
